\title{
Renal Actinomycosis, A Rare Diagnosis Which Can Clinically Mimic a Malignancy, Case Report and Review of the Literature
}

\author{
Nakisa Niknejad, ${ }^{1}$ Behnaz Moradi, ${ }^{2}$ Nasim Niknezhad, ${ }^{3}$ Masoomeh Safaei, ${ }^{1}$ and Fatemeh Nili1, \\ ${ }^{1}$ Department of Pathology, Cancer Institute, Imam Khomeini Hospital Complex, Tehran University of Medical Sciences, Tehran, IR Iran \\ ${ }^{2}$ Department of Radiology, Women's Yas Hospital, Tehran University of Medical Sciences, Tehran, IR Iran \\ ${ }^{3}$ Skin Research Center, Shahid Beheshti University of Medical Sciences, Tehran, IR Iran \\ "Corresponding author: Fatemeh Nili, Department of Pathology, Cancer Institute, Imam Khomeini Hospital Complex, Tehran University of Medical Sciences, Tehran, IR Iran. \\ Tel: +98-2161192504, Fax: +98-2166581526, E-mail: f-nili@sina.tums.ac.ir
}

Received 2017 May 12; Revised 2017 July 25; Accepted 2017 August 22.

\begin{abstract}
Introduction: Actinomycosis is an unusual infection, which usually appears in the thoracic, cervicofacial, and abdominal areas. Renal actinomycosis is rare and has not yet been reported in children.

Case Presentation: An 8-year-old boy presented with a fever, weight loss, and flank pain two weeks after a sore throat. As the imaging studies were in favor of the malignant tumor of the right kidney, he underwent right radical nephrectomy. Pathologic study revealed Xanthogranulomatous pyelonephritis and actinomyces bacterial colonies.

Conclusion: Renal actinomycosis is difficult to diagnose due to its non-specific clinical findings and near similarity to other inflammatory and tumoral conditions. Early diagnosis is important for medical treatment and decrease of un-necessary surgery. Using auxiliary diagnostic methods can be helpful in this aspect. Percutaneous core needle biopsy or fine needle aspiration possesses higher accuracy and specificity in comparison with other methods.
\end{abstract}

Keywords: Renal, Actinomycosis, Childhood

\section{Introduction}

Actinomycosis is a rare infection induced by Actinomyces bacteria. It clinically appears in the cervicofascial, thoracic, and abdominal areas as well as central nervous system (1). Abdominal-pelvic actinomycosis accounts for $10 \%-20 \%$ of the cases, which is growing in frequency related to abdominal surgery (such as appendectomy), long term use of contraceptive intrauterine devices, poor oral hygiene, neoplasia, bowel perforation, trauma, implantation of foreign bodies, and perforated viscus (2-4). Renal Actinomycosis is rare with fewer than 25 reported cases in adult patients, since 1990. This uncommon infection is clinically difficult to diagnose due to its near resemblance to a number of other inflammatory processes and neoplastic conditions. As the disease responds to treatment with high doses of antibiotics, accurate diagnosis is essential to avoid unnecessary operations (5). Herein, we report a case of a renal actinomycosis in an 8-year-old boy who underwent nephrectomy due to mistake of primary diagnosis. This is the first report of this rare disease in children.

\section{Case Report}

An 8-year-old boy and the first child of family, from a village in northwest of Iran, was admitted to a local hos- pital with the complaints of fever, weakness, weight loss (7kg within 2 weeks) and flank pain without dysuria or other urinary symptoms. He had a history of a sore throat, which had been improved without intervention two weeks ago. He was burned at 39 weeks of gestation by natural delivery and had a normal growth during infancy and after that. There was no significant disease on his past medical history. No important disease was mentioned in his family history. Physical examination at the time of admission revealed fever (38.2 oral temperature), tachycardia (puls rate 103/minute), and tenderness at costovertebral junction. On urine analysis, white blood cell (WBC) was positive (30 - 40/HPF) and bacteria negative. Urine culture had not been performed. Erythrocyte Sedimentation Rate (ESR) was $112 \mathrm{~mm} / \mathrm{hr}$. Leukocytosis with shift to left $\left(20.32 \times 10^{3} \mu \mathrm{l}\right)$ and anemia $(\mathrm{Hb} 9.9 \mathrm{~g} / \mathrm{dl})$ were detected on cell blood count. On ultrasonography, a solid cystic mass with extension to perinephric adipose tissue was identified. Pre-contrast computed tomography (CT) revealed enlargement of right kidney with Gerota's fascia thickening. There was no evidence of calculi. On postcontrast CT scan, a $74 \times 53 \times 48 \mathrm{~mm}$ heterogeneous mass with cystic component was identified in the upper pole of the right kidney. This mass had exophytic extension into peri-renal fat space (Figure 1). Based on these imaging find- 
ings, a possible neoplasm such as Wilms' tumor was suggested and right nephrectomy was done. No pathologic finding was detected in the liver or lung on pre-surgical metastatic work-up. On Gross pathologic examination, fragile heterogeneous grayish mass with irregular border measuring $65 \times 55 \times 25 \mathrm{~mm}$ was seen in the upper and middle pole of the right kidney. On microscopic examination, collections of histiocytes, foamy macrophages, polymorphonuclear leukocytes, and foci of micro-abscess formation were identified (Figure 1A). Scattered basophilic masses with eosinophilic terminal clubs (so-called sulfur granules) were dispersed between inflammatory cells (Figure 2B). Gram and Gomory-Methenamine-Silver stains revealed branching filamentous Gram-positive and silver positive rods at the periphery of the granules (Figure $2 \mathrm{C}$ and 2D, respectively). Acid fast special stain was negative. The final diagnosis was highly suggestive of Actinomyces xanthogranulomatous pyelonephritis. After surgery, body temperature decreased and the patient was discharged on the order of 7.5cc oral Cefalexin every night. Based on pathology diagnosis and after consultation with another center, the treatment was changed to amoxicillin, orally, three times a day for 4 months. Due to poor oral hygiene, the patient was referred for dental examination. Two decayed teeth were identified and extracted. Now, after a 10month follow up, the boy is well with appropriate weight gain and good general condition.

\section{Discussion}

In humans, actinomycosis is mostly caused by Actinomyces Israelii species (6). The agent of this disease is endogenous and normal flora of mouth, around the gums, teeth, crypts of tonsils, respiratory system, and gastrointestinal tract. Actinomycosis is implicated in the loss of host's mucosal barriers and infiltration of bacteria into the adjacent organ or bloodstream. It is characterized as chronic granulomatous and suppurative inflammation, which is usually complicated with abscess formation or sinus tract with sulfur granule discharge (7). Kidney involvement via direct extension from the contiguous structures or hematogenous infection is rare and reported in less than 25 studies since 1990, in English literature (Table 1) $(5,7-27)$.

Most of the reported cases have been in the right kidney, in men, and in patients older than 25 years. None of the cases were in children. Wong et al., (2011), showed that age and gender are two risk factors in an incidence of actinomycosis, as the incidence of disease is higher in men (28). The present report is regarding an 8-year-old-boy with right kidney involvement. This is the first report on a patient younger than 16 years old; however, the other two factors are consistent with the previous studies for gender and side of involvement.

Most of the patients were immunocompetent people without underlying diseases that were presented with non-specific clinical findings including abdominal pain, weight loss, fever, weakness, and overnight sweating, in the order of frequency, respectively. Hematuria and urinary symptoms were the other infrequent findings. Laboratory findings were also non-specific including anemia, leukocytosis, and elevated ESR.

With regards to this note that clinical signs and laboratory findings can not be helpful in definite diagnosis, clinicians use auxiliary methods such as culture and imaging for differential diagnosis. Actinomyces Spp. slowly grow on blood agar media at 35 centigrade degree for 7 days. According to previous reports, the results are negative in $76 \%$ of cases (29). Wagenlehner and coworkers showed in their study that the microbiology results could not be helpful in diagnosis of Actinomycosis (29). Gram staining and pathologic assessment of pus or infected tissue are more sensitive than microbial culture and are of great value for the diagnosis. On histopathologic examination, granulomatous inflammation, abscess, necrosis, and one to three sulfur granules could be identified in about $75 \%$ of the cases. These findings are highly suggestive but not specific for the diagnosis (1). Nocardiosis, Botriomycosis, and Chromomycosis are the main differential diagnoses (28). However, gram staining can show branching filamentous Gram-positive rods at the periphery of the granules, which is highly suggestive of actinomycosis. Nocardia can be usually differentiated from Actinomyces bacteria as they are partially Acid fast positive. Immunofluorescence study is highly specific in the diagnosis. Nowadays, molecular studies, such as 16S rRNA sequencing, are a reference method for identification and classification. Polymerase Chain Reaction (PCR), with specific primers, could be used for identification of microbial agents in clinical material (1). Unfortunately, we couldn't perform these complementary tests for our case. On the other hand, ultrasonography and CT scan are not diagnostic. As is shown in Table 1, in most of the cases, in imaging, actinomycosis presented as a mass like lesion (not infiltrative process) and mimic a malignant neoplasm, owing to its slow growth rate. Our case was also misdiagnosed as Wilm's Tumor based on imaging findings and young age of the patient. However in cases with a non-mass pattern, the presenting CT scan features can be cystic lesions with thickened walls and septa or with the infiltrating pattern like as the presence of sinus tract. In this situation, inflammatory reactions in adjacent structures like as the retroperitoneum and psoas muscle are frequent. With this pattern the renal tumor is an unlikely diagnosis $(4,7,20,23)$. A CT 

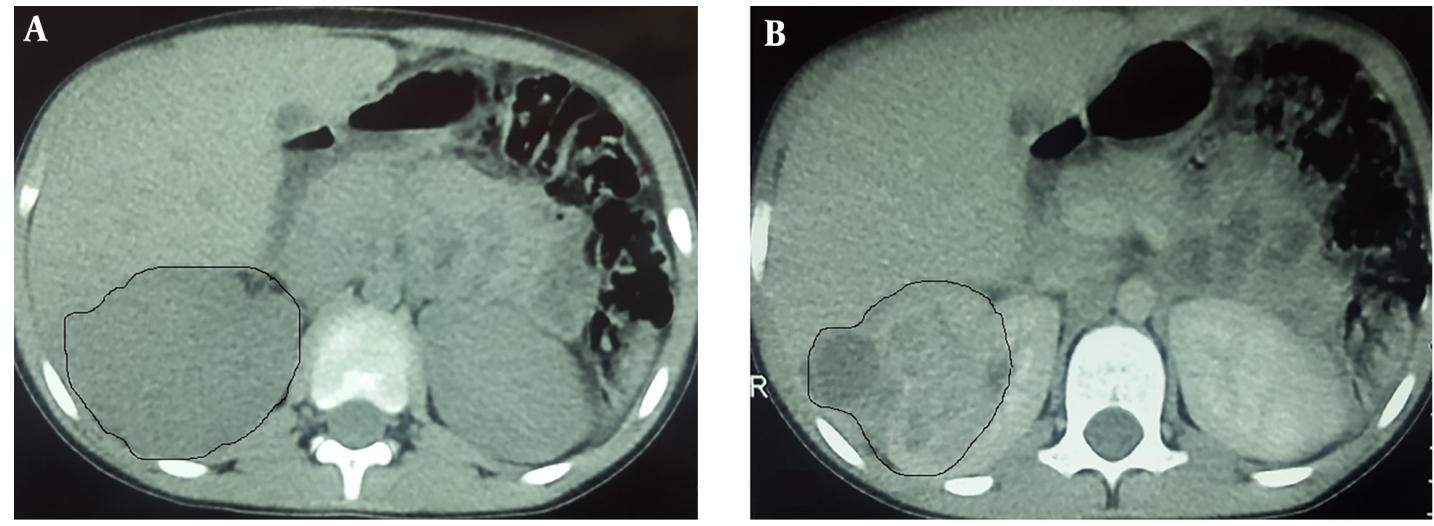

Figure 1. A, Precontrast CT scan shows decreased density and enlargement of right kidney; B, Gerota's fascia thickening is evident; C: Contrast enhanced CT scan in nephrogram phase showing a heterogeneous ill-defined mass with some cystic components and perinephric extension.
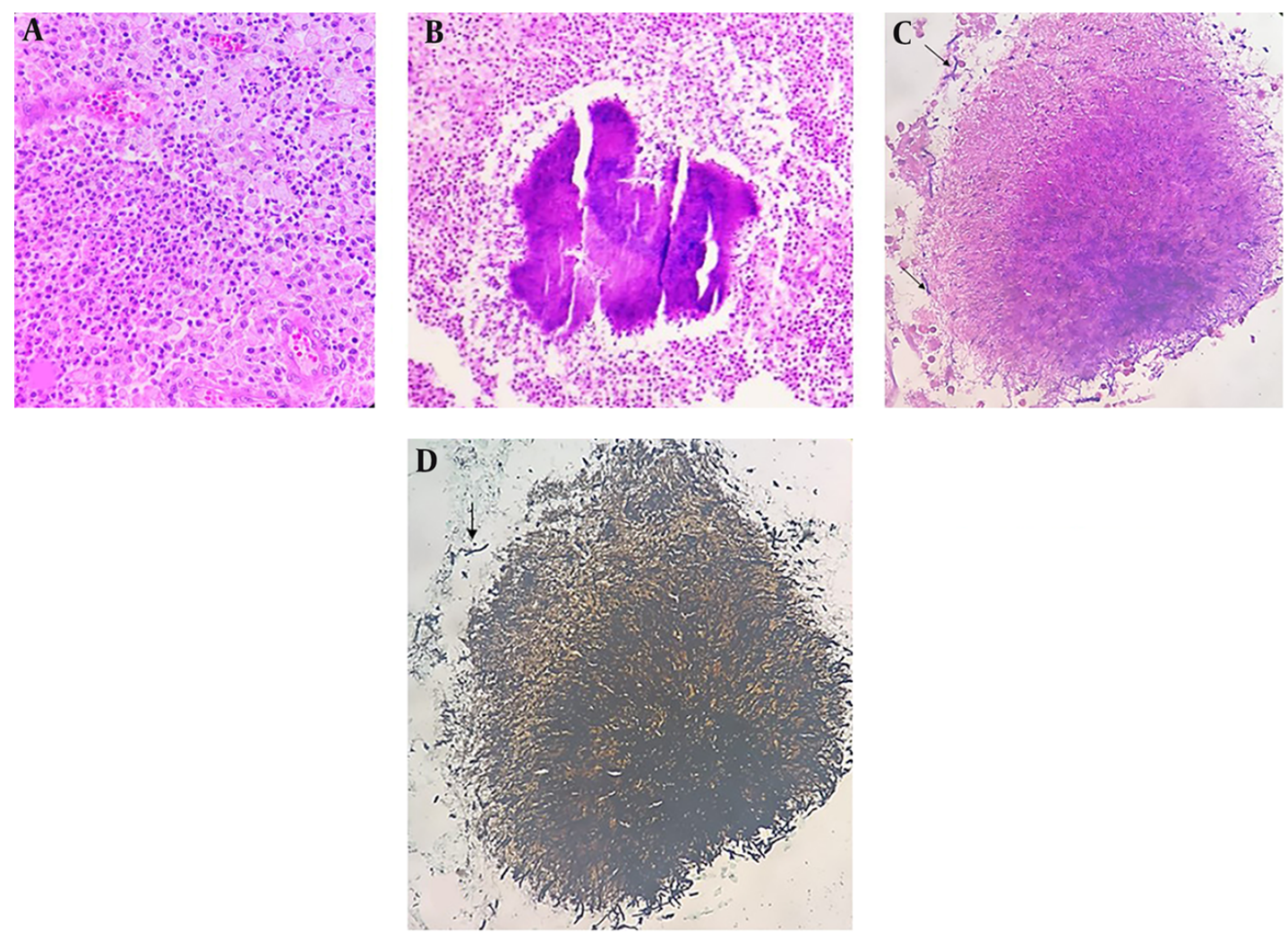

Figure 2. A, H\&E stained sections show collection of histiocytes, foamy macrophages, abscess and B, sulfur granules highly suggestive of actinomyces bacterial colonies; C, gram stain.

scan is usually useful for evaluation of the extent of the disease and involvement of adjacent organs. In some studies, the authors suggest that a MRI can be helpful to distinguishing actinomycosis from other differential diagnosis, especially malignant lymphoma. Actinomycosis show iso to low signal intensity on $\mathrm{T} 1$ and low signal intensity on T2-weighted sequences, whereas malignant lymphoma is characterized as iso-signal to high signal mass lesion (15). Manasanch and coworkers expressed in their report that sono-guided percutaneous needle aspiration or biopsy is 


\begin{tabular}{|c|c|c|c|c|c|c|c|}
\hline Number & Author (s) & Year & Age,y & Sex & Side & Imaging Findings & Risk Factor \\
\hline $\mathbf{1}$ & Cvetkov et al. (8) & 1995 & 19 & Female & Left & Infiltrative & Diabetes Mellitus \\
\hline 2 & Khalaff et al. (9) & 1995 & 62 & Male & Left & Tumorous & No \\
\hline 3 & Hyldgaard-Jensen et al. (10) & 1999 & 45 & Male & Right & Infiltrative & No \\
\hline 4 & Fujita et al. (11) & 1999 & 63 & Male & Right & Tumorous/ inflammatory mass & No \\
\hline 6 & Dieckmann et al. (13) & 2001 & 52 & Male & Left & Tumorous & Poor dental hygiene \\
\hline 7 & Hilfiker ML (14) & 2001 & 16 & Male & Right & Tumorous & Poor dental hygiene \\
\hline 8 & Horino et al. (15) & 2004 & 68 & Male & Right & Tumorous & No \\
\hline 9 & Dhanani et al. (16) & 2004 & 64 & Male & Right & Tumorous & No \\
\hline 10 & Gidwani et al. (17) & 2005 & 41 & Male & Left & Tumorous & No \\
\hline 11 & Sallami et al. (18) & 2006 & 25 & Female & Right & Tumorous & Pregnancy (May have been trigger) \\
\hline 13 & Yenarkarn et al. (20) & 2007 & 39 & Male & Left & Infiltrative & $\begin{array}{c}\text { Exploratory laparatomy and gastric } \\
\text { surgery }\end{array}$ \\
\hline 14 & Efthimiou et al. (21) & 2008 & 50 & Female & Left & Cystic & No \\
\hline 15 & Lin YH et al. (4) & 2009 & 42 & Female & Right & $\begin{array}{l}\text { Emphysematous pyelonephritis and } \\
\text { renal stones }\end{array}$ & Diabetes Mellitus \\
\hline 16 & Baburaj et al. (22) & 2009 & 55 & Male & Left & Tumorous & No \\
\hline 17 & Manasanch et al. (23) & 2012 & 80 & Female & Right & Tumorous & $\begin{array}{l}\text { Laparascopic cholecyctectomy and } \\
\text { left nephrectomy }\end{array}$ \\
\hline 18 & Lin C-D et al. (7) & 2012 & 59 & Female & Right & Cystic & Diabetes Mellitus \\
\hline 19 & Pourmand et al. (24) & 2012 & 56 & Male & Right & Renal Stone & $\begin{array}{l}\text { Uremic patient } \\
\text { (Immunocompromised) }\end{array}$ \\
\hline 20 & Agrawal et al. (25) & 2014 & 52 & Male & Right & Cystic & Diabetes Mellitus \\
\hline 21 & Smid MC et al. (26) & 2014 & 20 & Female & Right & Tumorous & $\begin{array}{l}\text { Laparascopic appendectomy and } \\
\text { cessarian section }\end{array}$ \\
\hline
\end{tabular}

preferred for diagnosis of actinomycosis and more effective in comparison with other imaging methods (23).

Accurate diagnosis is essential for choosing appropriate treatment modality and subsequent successful treatment outcome. Generally, actinomyces species are susceptible to Beta-lactam antibiotics and penicillin. The traditional concept of long time treatment with high doses of antibiotics is changing. Optimal duration of treatment depends on the site of involvement, performance of surgery and initial burden of the disease $(1,28)$. Surgery and invasive procedures are considered for the cases with extensive necrosis or failure in antibiotic therapy. The rate of mortality of actinomycosis, according to the site of involvement, time of diagnosis, and time of treatment beginning, was reported between $0 \%$ to $28 \%$. As a result, an early and accurate diagnosis is crucial for gaining the best medical care (28).

\subsection{Conclusion}

Actinomycosis is a rare cause of xanthogranulomatous pyelonephritis. Individuals of any age from 8-80 years could be involved. Most of the patients are immunocompetent people, presenting with non-specific clinical and laboratory findings. It is usually misdiagnosed as malignant neoplasm on imaging studies. Accurate diagnosis is essential for early medical treatment and avoiding unnecessary surgery.

\section{References}

1. Valour F, Senechal A, Dupieux C, Karsenty J, Lustig S, Breton P, et al Actinomycosis: etiology, clinical features, diagnosis, treatment, and management. Infect Drug Resist. 2014;7:183-97. doi: 10.2147/IDR.S39601. [PubMed: 25045274]. [PubMed Central: PMC4094581].

2. Pusiol T, Morichetti D, Pedrazzani C, Ricci F. Abdominal-pelvic actinomycosis mimicking malignant neoplasm. Infect Diseas Obstetric Gynecol. 2011;2011.

3. Garner JP, Macdonald M, Kumar PK. Abdominal actinomycosis. Int J Surg. 2007;5(6):441-8. doi: 10.1016/j.ijsu.2006.06.009. [PubMed: 18078685].

4. Lin Y-H, Chen H-W, Chuang C-K, Ng K-F, Wang H-H. Renal actinomycosis presented as emphysematous pyelonephritis: a case report. J Taiwan Urol Assoc. 2009;20:181-3.

5. Nunoo-Mensah JW, Joglekar VM, Nasmyth GD, Partridge SM. Abdominal actinomycosis-can early diagnosis prevent extensive surgery? Int J Clin Pract. 2010;64(1):106-9. doi: 10.1111/j.1742-1241.2006.00935.x. [PubMed: 20089023]. 
6. Chi Y, Liu X, Li G. Case Report Urachal actinomycosis mimicking carcinomatosis: a case report and review of literature. Int J Clin Exp Pathol. 2016;9(2):2575-8.

7. Lin C-D, Tai H-C, Wang C-C, Yu C-T, Chang S-J, Hsieh C-H. Renal actinomycosis-An unusual cause of a renal abscess. Urological Sci. 2012;23(4):129-32.

8. Cvetkov M, Elenkov C, Georgiev M, Topov U, Stefanova G. Renal actinomycosis complicated by renoduodenal fistula and diabetes mellitus. BJU Int. 1995;75(1):104-5.

9. Khalaff H, Srigley JR, Klotz LH. Recognition of renal actinomycosis: nephrectomy can be avoided. Report of a case. Can J Surg. 1995;38(1):77-9. [PubMed: 7882215].

10. Hyldgaard-Jensen J, Sandstrom HR, Pedersen JF. Ultrasound diagnosis and guided biopsy in renal actinomycosis. $\mathrm{Br} J$ Radiol. 1999;72(857):510-2. doi: 10.1259/bjr.72.857.10505021. [PubMed 10505021].

11. Fujita Y, Hirata Y, Hoshino T, Nasu N, Imagawa M, Nakagawa M. [A case of renal actinomycosis]. Nihon Hinyokika Gakkai zasshi. Japan JUrolog. 1999;90(10):843-6.

12. Horvath K, Porkolab Z, Palko A. Primary renal and retroperitoneal actinomycosis. Eur Radiol. 2000;10(2):287-9. doi: 10.1007/s003300050045. [PubMed: 10663758].

13. Dieckmann KP, Henke RP, Ovenbeck R. Renal actinomycosis mimicking renal carcinoma. Eur Urol. 2001;39(3):357-9. doi: 10.1159/000052467. [PubMed: 11275733].

14. Hilfiker ML. Disseminated actinomycosis presenting as a renal tumor with metastases. J Pediatr Surg. 2001;36(10):1577-8. doi: 10.1053/jpsu.2001.27053. [PubMed: 11584412].

15. Horino T, Yamamoto M, Morita M, Takao T, Yamamoto Y, Geshi T. Renal actinomycosis mimicking renal tumor: case report. South Med J. 2004;97(3):316-8. doi:10.1097/01.SMJ.0000072362.01568.D7. [PubMed: 15043347].

16. Dhanani NN, Jones DM, Grossman HB. Medical management of renal actinomycosis. J Urol. 2004;171(6 Pt 1):2373-4. [PubMed:15126826].

17. Gidwani AL, Connolly D, Khan A, Kemohan R, Brown M, Kenny B. Renal, colonic and retroperitoneal actinomycosis-a case report. West Afr J Med. 2005;24(4):343-5. [PubMed: 16483055].

18. Sallami S, Nouira Y, Hmidi M, Mekni A, Hendaoui L, Horchani A.
Pseudo-tumoral renal actinomycosis. Int J Urol. 2006;13(6):803-4. doi: 10.1111/j.1442-2042.2006.01407.x. [PubMed:16834665].

19. Jalon Monzon A, Alvarez Mugica M, Seco Navedo MA, Fernandez Gomez JM, Bulnes Vazquez V, Gonzalez Alvarez RC. A new form of presentation of renal actinomycosis: renal tumor with retroperitoneal bleeding. Archivos Espa-oles de Urologla (Ed impresa). 2006;59(7):756-

20. Yenarkarn P, Thoeni RF, Hanks D. Case 117: actinomycosis of left kidney with sinus tracts. Radiology. 2007;244(1):309-13. doi: 10.1148/radiol.2441041211. [PubMed: 17581911].

21. Efthimiou I, Mamoulakis C, Petraki K, Zorzos I. Renal actinomycosis presenting as a suppurated solitary cyst. Indian JUrol. 2008;24(3):4168. doi: 10.4103/0970-1591.42631. [PubMed: 19468482]. [PubMed Central: PMC2684353].

22. Baburaj P, Antony T, Madhu C, Krishnan HB. A rare case of pyrexia of unknown origin due to renal actinomycosis. Kerala Med $\mathrm{J}$. 2009;2(3):91-2.

23. Manasanch EE, Grivas PD, Walter O, Davaro R. Kidney Actinomycosis, A Rare Diagnosis That Can Mimic Renal Cell Carcinoma: A Case Report and Review of the Literature. Infectious Diseas Clinical Practic. 2012;20(5):362-4.

24. Pourmand M, Dehghani S, Hadjati M, Kosari F, Pourmand G. Renal Actinomycosis in Presence of Renal Stones in a Patient with End Stage Renal Disease. J Med Bacteriol. 2012;1(3-4):62-5.

25. Agrawal P, Vaiphei K. Renal actinomycosis. BMJ Case Report. 2014;2014:bcr2014205892.

26. Smid MC, Bhardwaj NR, Di Giovanni LM, Eggener S, Torre MD. Renal hemorrhagic actinomycotic abscess in pregnancy. Infect Dis Rep. 2014;6(1):5157. doi: 10.4081/idr.2014.5157. [PubMed: 24757509]. [PubMed Central: PMC3987246].

27. Gulwani HV. Xanthogranulomatous pyelonephritis with colesional actinomycosis in a 63-year-old man. Indian J Urol. 2015;31(2):144-5. doi: 10.4103/0970-1591.152925. [PubMed: 25878419]. [PubMed Central: PMC4397554].

28. Wong VK, Turmezei TD, Weston VC. Actinomycosis. BMJ. 2011;343:d6099. doi: 10.1136/bmj.d6099. [PubMed: 21990282].

29. Wagenlehner FM, Mohren B, Naber KG, Mannl HF. Abdominal actinomycosis. Clin Microbiol Infect. 2003;9(8):881-5. [PubMed: 14616714]. 